Diabetologe 2009 · 5:416-417

DOI 10.1007/s11428-009-0474-1

Online publiziert: 29. August 2009

(c) Springer Medizin Verlag 2009

\author{
W.A. Scherbaum \\ Klinik für Endokrinologie, Diabetologie und Rheumatologie, \\ Universitätsklinikum Düsseldorf
}

\title{
Sicherheit von Insulin Glargin
}

den Ängsten der besorgten Patienten umzugehen.

Ausgangspunkt war eine AOK-Studie mit dem Leiter des Instituts für Qualität und Wirtschaftlichkeit im Gesundheitswesen (IQWiG) als Seniorautor, in der behauptet wurde, dass Insulin Glargin mit einem erhöhten Krebsrisiko verbunden sei [2]. ropean Medicines Agency“ (EMEA), offiziell bekundet [1], was bereits zuvor mehrere Gremien von wissenschaftlichen Fachgesellschaften (ADA, EASD, DDG), besetzt mit führenden Wissenschaftlern, sowie die amerikanische Zulassungsbehörde FDA erarbeitet hatten:

„Die verfügbaren Daten geben keinen Grund für Besorgnis bezüglich der Sicherheit von Insulinanaloga, speziell des langwirksamen Insulinanalogs Glargin (Lantus $^{\oplus}$, Optinsulin $\left.{ }^{\oplus}\right)$. Die vorliegenden Daten erlauben weder eine Beziehung zwischen der Anwendung von Insulin Glargin und Krebs herzustellen noch eine solche auszuschließen."

Einige Vertreter der Sensationspresse, sogar der öffentlich-rechtlichen Sender, haben sich als Wissenschaftler versucht und aus dem Für und Wider in der Diskussion der Zellkulturversuche, der tierexperimentellen und den klinischen Daten die ihnen gefälligen Argumente und Sätze herausgepickt und ein Bild von den vermeintlich leichtfertig agierenden Ärzten gezeichnet. Das ist, gelinde gesagt, haarsträubend.

Ist der Schaden wieder gutzumachen? Ich meine nicht die Verkaufszahlen von Insulinanaloga, sondern den Vertrauensverlust für die Ärzte. Für die Sensationspresse ist die Story vorbei; die Ärzte haben nun aber die langwierige Aufräumarbeit an der Basis zu bewältigen und mit

\section{- Ein im Lancet publizierter \\ Kommentar [3] zur mangelhaften methodischen Qualität dieser Studie kommt einer Ohrfeige für den Leiter des IQWiG gleich.}

Bei einer mittleren Beobachtungsdauer von 1,63 Jahren zeigte sich eine Assoziation zwischen der Gabe von Insulin (unabhängig vom Insulintyp) und dem Krebsrisiko. Patienten mit Kombinationen aus verschiedenen Insulinen waren ausgeschlossen worden. Es zeigte sich, dass die Verschreibung von Glargin mit einem geringeren Krebsrisiko verbunden war als Humaninsulin. Durch nachträgliche statistische Anpassungen wurde die Aussage jedoch umgekehrt. Auch gab es in der Studie keine Angaben zum „Body Mass Index" und zu der Art der Tumorerkrankungen im Register.

Um die mögliche Beziehung zwischen der Behandlung mit Insulinanaloga und Tumoren weiter aufzuklären, wurden Wissenschaftler aus Schweden, Schottland und England gebeten, die Daten aus ihren Registern auf diese Frage hin zu untersuchen. Die Ergebnisse waren äußerst widersprüchlich: Die schwedische Studie fand kein erhöhtes Karzinomrisiko bei Lantus ${ }^{\circ}$ gegenüber Humaninsulin [4]. Die schottische Studie fand ein leicht reduziertes Karzinomrisiko unter Lantus ${ }^{\oplus}$ im Vergleich zu Humaninsulin; die Gruppe mit alleiniger Lantus ${ }^{\circ}$-Therapie hatte etwas häufiger Karzinome, sie war aber auch deutlich älter als die Vergleichsgruppe [5]. Die englische Registerstudie fand keinen Unterschied des Karzinomrisikos zwischen verschiedenen Insulinen [6]. Zu jeder der Registerstudien gab es jeweils noch verschiedene Subanalysen.

Einige frühere retrospektive epidemiologische Studien haben in der Tat eine Assoziation zwischen Karzinomen und der Anwendung von Insulin gezeigt [7, 8, 9]. Karzinome kommen aber auch im höheren Lebensalter, bei Adipositas und bei Insulinresistenz gehäuft vor. Die retrospektive Interpretation von Registerdaten ist auch dadurch erschwert, dass bei verschiedenen Patientengruppen verschiedene Insulinstrategien über eine unterschiedlich lange Zeit angewandt werden. Noch fragwürdiger ist sind Interpretationen zur Sicherheit einzelner Insuline aus solchen Registerdaten, die aus nicht dafür angelegten und nicht entsprechend stratifizierten Populationen extrahiert werden.

\section{(2) Das IQWiG muss bei der Beurteilung von klinischen Daten unabhängig sein}

An dieser Stelle muss an die Verantwortung des IQWiG appelliert werden, das ja unabhängig tätig sein sollte. Die Beurteilung von klinischen Daten muss unabhängig und nach strengen wissenschaftlichen Kriterien erfolgen. Dies trifft nicht nur für Ärzte, sondern insbesondere auch für die Aufsichtsbehörden zu, die die Anwendung von Arzneimitteln überwachen und z. T. reglementieren. Im vorliegenden 
Falle war jedoch der Leiter des IQWiG in die ausschlaggebende Registerstudie des AOK-abhängigen WIdO (Wissenschaftliches Institut der AOK) involviert, und es blieb bei den meisten Statements in o. g. Sache unklar, ob er denn seine eigene Interpretation als Autor oder die des IQWiG wiedergibt. Letzteres hätte nur nach Prüfung durch eine unabhängige Expertenkommission erfolgen dürfen. Diese Interessenverflechtung und auf eine Person konzentrierte Struktur des IQWiG ist ein alt bekanntes und bisher nicht gelöstes Problem.

\section{Konsequenzen}

Was sind die Konsequenzen?

\section{Für die klinische Praxis}

Es gibt von wissenschaftlicher Seite - auch auf der Basis der 4 in der Zeitschrift Diabetologia publizierten Registerstudien - keinen Grund, Insulinanaloga, speziell Glargin (Lantus ${ }^{\oplus}$, Optinsulin ${ }^{\circledast}$ ), abzusetzen bzw. auf ein anderes Insulin umzustellen.

Sollten Patienten jedoch durch die vorausgegangenen Diskussionen zur Sicherheit von Insulinanaloga trotz der Aufklärung über den Sachverhalt noch verunsichert sein, so sollte man ihnen durchaus anbieten, die Insulintherapie umzustellen.

\section{Aufklärung der Patienten}

Jeder Arzt, der Patienten mit Diabetes behandelt, sollte dazu beitragen, die Patienten bezüglich der Krebsangst bei der Anwendung von Insulin zu beruhigen und sie über den richtigen Sachverhalt aufklären.

\section{Appell an die Medien}

Die Medien sollten dazu aufgefordert werden, die Nachricht über den fehlenden wissenschaftlich begründeten Zusammenhang zwischen Glargin und Krebs zu verbreiten. Alle Kolleginnen und Kollegen, die Zugang zu Medien haben, sollten versuchen, zu dieser Klarstellung beizutragen.
Erforschung des Zusammenhangs zwischen Insulin und Krebsrisiko

Die Aufgabe der Forschung ist es nun, wissenschaftliche Studien aufzulegen, die spezifisch dazu angelegt und in der Lage sind, den Zusammenhang zwischen der Anwendung von Insulin im Allgemeinen und von Insulinanaloga wie Glargin im Besonderen und dem Auftreten von Karzinomen aufzuklären. Die EMEA hat die Firma Sanofi-Aventis dazu aufgefordert, eine Strategie für solche Studien aufzuzeigen. Ein Expertenkomitee der Firma schlug nun vor, als Zwischenschritt zunächst einmal die bisher verfügbaren einzelnen oder gepoolten Daten von randomisierten klinischen Studien mit geeigneten statistischen Anpassungen für Karzinomrisiko auszuwerten und $\mathrm{zu}$ publizieren. Auch wenn dies noch nicht einer spezifisch dafür angelegten Studie entspricht, so sind solche Daten den Registerdaten haushoch überlegen.

\section{(2) Daten aus randomisierten klinischen Studien sind Registerdaten weit überlegen}

In einer kürzlich publizierten prospektiven randomisierten klinischen Studie zur retinalen Sicherheit von Glargin wurden z. B. 1024 Patienten mit Typ-2-Diabetes entweder mit Glargin oder mit NPH-Insulin behandelt und mit einer mittleren Expositionsdauer von 4 Jahren für das entsprechende Insulin untersucht. Wenn die Studie auch nicht für Karzinomrisiken stratifiziert war, so zeigte sich jedoch bei einer Post-hoc-Analyse, dass unter Insulin Glargin die Zahl der Karzinome nicht höher war als mit Humaninsulin [10, 11].

\section{Die Rolle der}

\section{Versorgungsforschung}

Insgesamt bleibt die Frage zu klären, wie man Daten aus der Versorgung zuverlässig analysieren kann. Tatsächlich können seltene Nebenwirkungen und auch in den speziellen Zulassungsstudien für Arzneimittel nicht berücksichtigte Zusammenhänge erst dann gesehen werden, wenn diese Medikamente bei vielen Patienten angewandt werden. Daher müssen alle Medikamente auch nach ihrer Zulassung regelmäßig auf den Prüfstand. Es ist jedoch dringend er- forderlich, Standards für eine zuverlässige Analyse von Versorgungsdaten zu erarbeiten und nach dem höchsten wissenschaftlichen Maßstäben auszurichten.

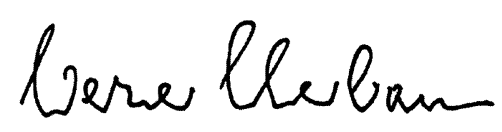

W. A. Scherbaum

\section{Korrespondenzadresse \\ Prof. Dr. W. A. Scherbaum}

Klinik für Endokrinologie, Diabetologie und Rheumatologie, Universitätsklinikum Düsseldorf Moorenstr. 4, 40225 Düsseldorf

scherbaum@uni-duesseldorf.de

Interessenkonflikt. Der korrespondierende Autor weist auf folgende Beziehungen hin: Der Autor ist Mitglied internationaler Advisory Boards der Firmen Sanofi-Aventis, Lilly, Pfizer, Novartis, AstraZeneca.

\section{Literatur}

1. EMEA, European Medicines Agency update on safety of insulin glargine. http://www.emea.europa. eu/humandocs/PDFs/EPAR/Lantus/47063209en. pdf (Press Release vom 23. Juli 2009)

2. Hemkens LG, Grouven U, Bender R et al (2009) Risk of malignancies in patients with diabetes treated with human insulin or insulin analogues: a cohort study. Diabetologia 52:1732-1744

3. Pocock SJ, Smeath L (2009) Insuline glargine and malignancy: an unwarranted alarm. Lancet [Epub July 20]

4. Jonasson JM, Ljung R, Talbäck M et al (2009) Insulin glargine use and short-term incidence of malignancies-a population-based follow-up study in Sweden. Diabetologia 52:1745-1754

5. Colhoun HM, SDRN Epidemiology Group (2009) Use of insulin glargine and cancer incidence in Scotland: A study from the Scottish Diabetes Research Network Epidemiology Group. Diabetologia 52:1755-1765

6. Currie CJ, Poole CD, Gale EAM (2009) The influence of glucose-lowering therapies on cancer risk 5 in type 2 diabetes. Diabetologia 52:1766-1777

7. Larsson SC, Mantzoros CS, Wolk A (2007) Diabetes mellitus and risk of breast cancer: a meta-analysis. Int J Cancer 121:856-862

8. Larsson SC, Orsini N, Wolk A (2005) Diabetes mellitus and risk of colorectal cancer: a meta-analysis. J Natl Cancer Inst 97:1679-1687

9. Huxley R, Ansary-Moghaddam A, Berrington de González A et al (2005) Type-Il diabetes and pancreatic cancer: a meta-analysis of 36 studies. $\mathrm{Br} \mathrm{J}$ Cancer 92:2076-2083

10. Rosenstock J, Fonseca V, McGill JB et al. (2009 a) Similar progression of diabetic retinopathy with insulin glargine and neutral protamine Hagedorn (NPH) insulin in patients with type 2 diabetes: a long-term, randomized, open-label study. Diabetologia 52:1778-1788

11. Rosenstock J, Fonseca V, McGill JB et al (2009 b) Similar risk of malignancy with insulin glargine and neutral protamine Hagedorn (NPH) insulin in patients with type 2 diabetes: findings from a 5 year randomised open-label study. Diabetologia 52:1971-1973 